\section{Intraventrikuläre Blutung: Besseres Outcome mit Lyse und Lumbaldrainage}

\begin{abstract}
Die Prognose von Patienten mit intraventrikulärer Blutung hat sich in den vergangenen Jahren durch neue Therapieoptionen wie intraventrikuläre Fibrinolyse und Lumbaldrainage deutlich verbessert. Beide Verfahren werden derzeit allerdings noch nicht routinemäßig eingesetzt, sondern sind erfahrenen Zentren vorbehalten.
\end{abstract}

$B^{2}$ ei etwa jedem zweiten Patienten mit intrazerebraler Blutung (ICB) kommt es in der Folge auch zu Einblutungen in das Ventrikelsystem. Meist mit dramatischen Konsequenzen. Denn bei $15 \%$ bis $30 \%$ der Patienten verlegt das intraventrikuläre Blut den dritten und vierten Ventrikel, sodass sich ein lebensbedrohlicher obstruktiver Hydrozephalus ausbildet. Besonders häufig treten Ventrikelnachblutungen bei antikoagulierten Patienten auf.

\section{EVD oft nicht ausreichend}

„Eine Ventrikelblutung ist ein massiver negativer Prognosefaktor", betonte Privatdozent Dr. Dimitre Staykov vom Krankenhaus der Barmherzigen Brüder in Eisenstadt. Zum einen wird das Gehirn durch direkte Masseneffekte und den Anstieg des intrakraniellen Drucks (ICP) geschädigt, zum anderen durch toxische Blutabbauprodukte. Letztere können zur Arachnoiditis mit Ausbildung eines Hydrozephalus malresorptivus

führen. Um Druckanstieg und Liquorstau vorzubeugen, ist die notfallmäßige Anlage einer externen Ventrikeldrainage (EVD) indiziert. Bei vielen Patienten, etwa mit großen Hämorrhagien, sei dieses Vorgehen alleine jedoch nicht ausreichend und mit einem nicht unerheblichem Infektionsrisiko assoziiert. Patienten mit schweren Einblutungen profitieren nach Versiegen der Blutungsquelle deshalb häufig von der unter CT-Kontrolle durchgeführten intraventrikulären Lyse, zum Beispiel mit dem rekombinantem Plasminogenaktivator rt-PA. So löst sich das Ventrikelhämatom schneller auf, toxische Blutabbauprodukte werden rascher eliminiert. Wie Staykov betonte, ist die intraventrikuläre Lyse bisher allerdings nicht für die Routineanwendung geeignet, sondern wird nur in erfahrenen Zentren durchgeführt.

Dennoch entwickelt jeder dritte Patient mit einer Ventrikelblutung eine permanente Resorptionsstörung. „Hier gibt

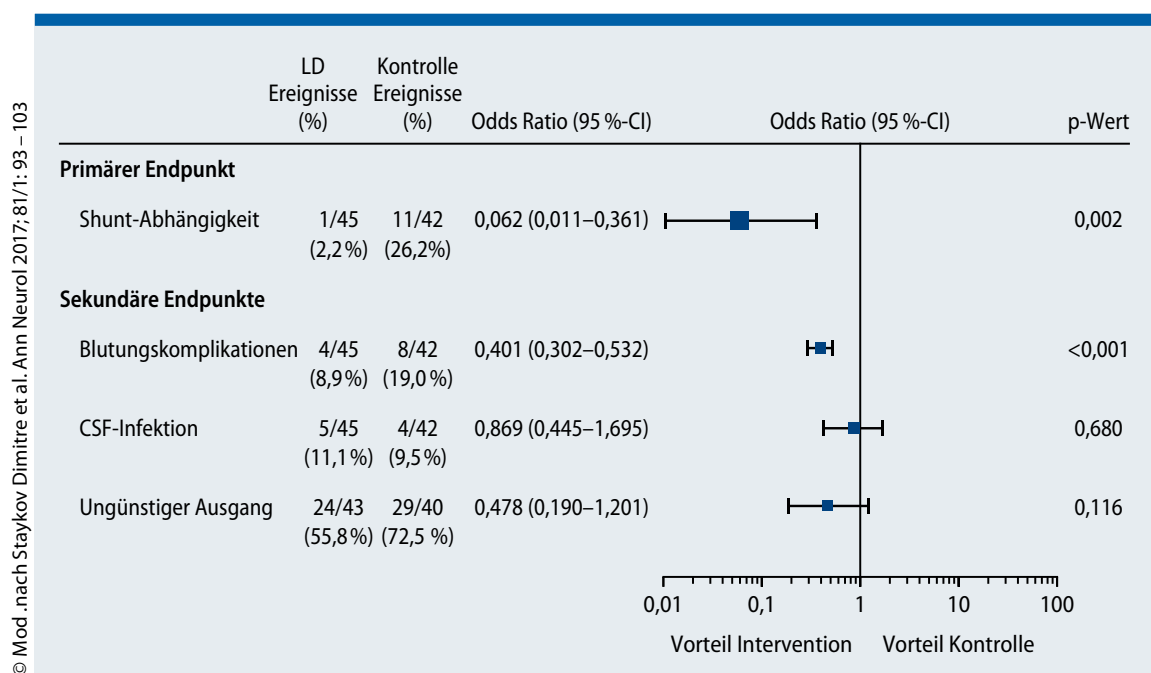

Abb. 1: Die kombinierte intraventrikuläre Fibrinolyse und frühe lumbale Drainage (LD) kann die Shunt-Implantation häufig vermeiden. es bisher nur wenige therapeutische Möglichkeiten“, so Staykov. Die meisten Patienten erhalten nach zwei bis drei Wochen einen ventrikulo-peritonealen (VP) Shunt. Die Shunt-Anlage sei aber oft unbefriedigend, wenn man die Malfunktionen und das Infektionsrisiko betrachtet. So müssen rund drei Viertel der Patienten revidiert werden.

\section{LD: Weniger Shunt-Implantationen nötig}

Scheitern die Abklemmversuche nach initialer EVD und Lyse, kann die Liquordrainage auch über den lumbalen Subarachnoidalraum fortgeführt werden. Die Lumbaldrainage (LD) hat den Vorteil, dass sie deutlich weniger invasiv ist, setzt aber voraus, dass dritter und vierter Ventrikel frei von Blut sind und eine Verbindung zwischen inneren und äußeren Liquorräumen besteht (Hydrozephalus communicans).

Dass man durch kombinierte Lyse und frühe LD die Shunt-Implantation häufig vermeiden kann, habe die Studie LUCAS-IVH (LUCAS-IVH = Lumbar Catheters in Severe IntraVentricular Hemorrhage) $(\mathrm{n}=30)$ gezeigt [Staykov Dimitre et al. Ann Neurol 2017, 81/1: $93-103]$. Sie untersuchte, wie häufig IVH-Patienten einen Shunt benötigen, wenn sie die Standardtherapie (EVD und Lyse) erhalten oder eine Kombination aus Lyse und LD (Abb. 1). Ergebnis: Während sieben der 16 EVD-Patienten einen Shunt benötigten, musste bei keinem der 14 LD-Patienten eine Shunt-Implantation vorgenommen werden. Im Vergleich zur EVD waren auch die Drainagezeiten bei allen LD-Patienten kürzer. Eine geringere Shunt-Abhängigkeit bei LD bestätigte sich darüber hinaus in einer Metaanalyse dreier vorangegangener Studien $(\mathrm{n}=97)$.

Als mögliche Ursachen für das bessere Outcome bei LD werden diskutiert: besserer Abtransport der toxischen Blutabbauprodukte, Prophylaxe einer schweren Arachnoiditis und Aufbau eines physiologischen Liquorgradienten.

Dr. Martina-Jasmin Utzt

Vortrag "Intraventrikuläre Fibrinolyse und lumbale Drainage - Ergebnisse einer randomisierten Studie und Metaanalyse", 90. DGN-Kongress, Leipzig, 23.9.2017 